\title{
Clinical and Microbiological Outcomes of Topical Aloe Vera Gel vs. Photochemotherapy as an Adjunct to Non-surgical Periodontal Treatment in Periodontitis
}

\section{(c) $(9)$}

Authors

Mohamed Farouk Elsadek¹, 2, Badreldin Mohamed Ahmed1, Rayan M. Eskandrani³, Tasneem Sobhy Fahmy²

\section{Retraction Note}

The authors have retracted this article.

received 21.03 .2020

revised $\quad 03.04 .2020$

accepted $\quad 15.04 .2020$

Bibliography

DOI https://doi.org/10.1055/a-1159-0738

Planta Med Int Open 2020; 7: e100-e105

(c) Georg Thieme Verlag KG Stuttgart · New York

ISSN 2509-9264

\section{Correspondence}

Mohamed Farouk Elsadek

Department of Community Health Sciences, College of

Applied Medical Sciences, King Saud University

Riyadh

Saudi Arabia

mfelsadek@gmail.com 